\title{
Assessment of Uncertainty in the Determination of Activation Energy for Polymeric Materials
}

\author{
Stephania P. Darby* \\ NASA Marshall Space Flight Center, Huntsville, Alabama 35812 \\ and \\ D. Brian Landrum $\dagger$ and Hugh W. Coleman $\ddagger$ \\ University of Alabama in Huntsville, Huntsville, Alabama 35899
}

\begin{abstract}
An assessment of the experimental uncertainty in obtaining the kinetic activation energy from thermogravimetric analysis (TGA) data is presented. A neat phenolic resin, Borden SC1008, was heated at three heating rates to obtain weight loss vs temperature data. Activation energy was calculated by two methods: the traditional Flynn and Wall method based on the slope of $\log (q)$ versus $1 / T$, and a modification of this method where the ordinate and abscissa are reversed in the linear regression. The modified method produced a more accurate curve fit of the data, was more sensitive to data nonlinearity, and gave a value of activation energy $75 \%$ greater than the original method. An uncertainty analysis using the modified method yielded a $60 \%$ uncertainty in the average activation energy. Based on this result, the activation energy for a carbon-phenolic material was doubled and used to calculate the ablation rate in a typical solid rocket environment. Doubling the activation energy increased surface recession by $3 \%$. Current TGA data reduction techniques that use the traditional Flynn and Wall approach to calculate activation energy should be changed to the modified method.
\end{abstract}

\section{Nomenclature}

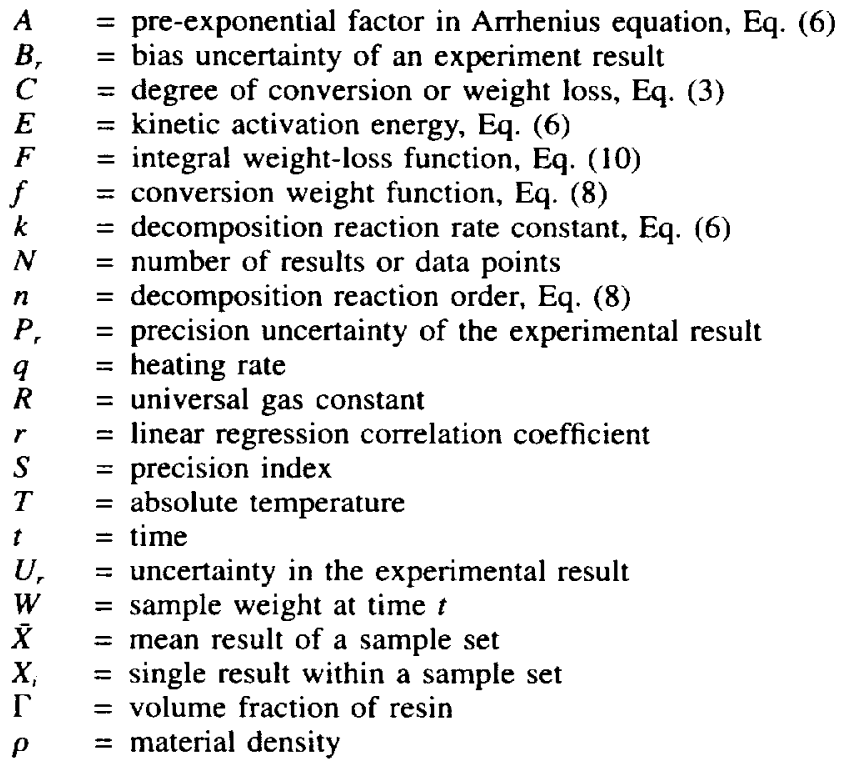

Received June 25, 1997; revision received Dec. 12, 1997; accepted for publication Dec. 13, 1997. Copyright (C) 1998 by the American Institute of Aeronautics and Astronautics. Inc. No copyright is asserted in the United States under Title 17, U.S. Code. The U.S. Government has a royalty-free license to exercise all rights under the copyright claimed herein for Governmental purposes. All other rights are reserved by the copyright owner.

*Materials Engineer, Team Leader, EH42. E-mail: stephania. darby@msfc.nasa.gov. Member AIAA.

†Assistant Professor. Propulsion Research Center, Department of Mechanial and Aerospace Engineering, RI E-33. Senior Member AIAA.

$\ddagger$ Eminent Scholar in Propulsion and Professor, Department of Mechanical and Aerospace Engineering, RI E-20. Associate Fellow AIAA.

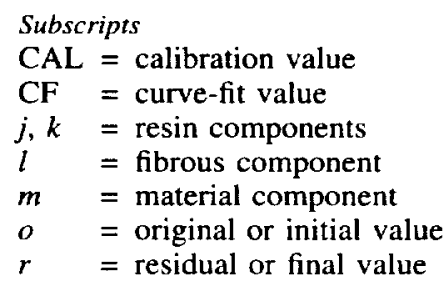

\section{Introduction}

$\mathbf{P}$ OLYMERIC and polymer-based composite materials are widely used in the aerospace industry for bonding, insulating, and sealing. A common application of these materials is in ablative cooling of combustion chambers and rocket nozzles. In this application a matrix of high-temperature-resistant fibers (such as carbon or silica) is impregnated with an organic phenolic or epoxy resin. The impregnated matrix is cure hardened into an appropriately shaped rigid structure. When exposed to the high-temperature characteristics of the burning propellant gases, the material undergoes a complex process combining chemical decomposition, melting, sublimation, charring, and evaporation. ${ }^{1-3}$ This physicochemical process of ablation causes the formation of a char layer on the surface, which is a poor heat conductor, thus forming a protective thermal blanket for the underlying structure. ${ }^{2,4}$

The Space Shuttle solid rocket motor (SRM) nozzle thermal protection system is composed of an ablative carbon-phenolic composite material. Current material research seeks to reduce the amount of composite required in the nozzle and thereby reduce nozzle weight (and thus launch costs). Conservative, i.e., thicker, ablative liners are often used because of the degree of uncertainty currently present in ablation simulation codes. Improved analytical techniques for predicting material ablation are thus required. ${ }^{5.6}$

Thermogravimetric analysis (TGA) ${ }^{7}$ is a technique particularly suited for quickly characterizing the thermal degradation of a material with time and/or temperature. Kinetic reaction parameters as well as weight fractions of material components are calculated from the TGA data and input to computational ablation simulation codes. As with any type of experimental 


\section{Assessment of Uncertainty in the Determination of Activation Energy for Polymeric Materials S. P. Darby, D. B. Landrum, H. W. Coleman}

Reprinted from

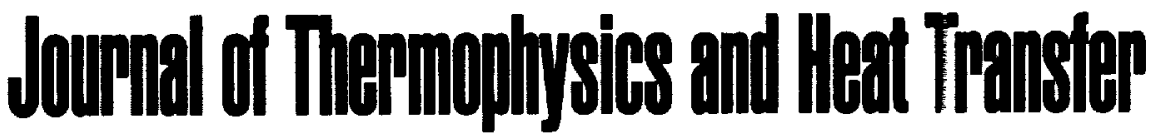

Volume 12, Number 4, Pages 520-527

\section{OAIAA}

A publication of the

American Institute of Aeronautics and Astronautics, Inc.

1801 Alexander Bell Drive, Suite 500

Reston, VA 20191-4344 
approach (and resulting calculations) some error is inherent in the process. Assessing the experimental uncertainty associated with the TGA process would lead to increased confidence in the resultant simulation-code predictions of nozzle ablation.

Many studies have been conducted that compare various methods of determining kinetic reaction parameters of polymeric materials from TGA data. ${ }^{8-12}$ These methods include curve fitting (using empirical TGA data and iteratively solving for reaction parameters), approximating the integral of the TGA decomposition equation, and obtaining kinetic parameters from integral weight fractions as a function of temperature. Although no consensus has been reached as to the most accurate method, ${ }^{13}$ the procedure developed by Flynn and $\mathrm{Wall}^{8,14}$ is widely used and has been incorporated into the data-reduction programs of several commercially available TGA instruments. This method uses linear regression analysis to determine the relationship between log heating rate and reciprocal temperature. The slope of the plot resulting from the regression equation is used to calculate activation energy.

Typically, kinetic reaction data are generated at low heating rates. Because extremely high heating rates are observed in actual rocket nozzles the generation of TGA data at these high rates are also being examined. ${ }^{15}$ The specialized equipment that has been developed for these studies is not commercially available. A comprehensive literature search has indicated that no one has systematically assessed the experimental uncertainty associated with the determination of kinetic reaction parameters by one particular methodology and commercially available instrument. In the study presented in this paper typical procedures for determining activation energy from TGA data were evaluated. The uncertainty analysis methods of Coleman and Steele ${ }^{16}$ were then employed to perform a detailed analysis of the propagation of uncertainty through the experimental determination of activation energy. The value of these methods for determining uncertainties associated with linear regression analysis has been demonstrated using Monte Carlo simulations. ${ }^{17}$

Most data for carbon-phenolic composites are restricted by the International Traffic in Arms Regulations. ${ }^{18}$ Because the purpose of this research was to assess the uncertainty in the experimental TGA methodology by examining typical procedures for determining activation energy, neat (no fibers) polymeric resin SC1008 was used. Without the effect of carbon fibers it is not expected that the activation energy of a neat phenolic resin would be the same as that of a phenolic composite. The uncertainty in activation energy of a composite may be greater than that of a neat resin because of errors caused by sample nonuniformity. Ten trials of the neat polymeric resin were conducted at each of three different heating rates. Based on the resultant calculated experimental uncertainty, standard activation energies for a carbon-phenolic material were increased by an equivalent factor and used to calculate the ablation rate in a typical solid rocket environment.

\section{Ablation Process}

Several computer codes have been written to predict the ablation rate of carbon-phenolic insulation materials in a rocket nozzle environment. The charring material thermal response and ablation program (CMA) code, and derivatives thereof, is currently used by NASA and its contractors for thermal analysis of the SRM ablative nozzle. The physicochemical ablation model is shown schematically in Fig. 1. Heating causes chemical decomposition in upper layers of the composite material, which results in the formation of a char layer at the top of a pyrolysis zone. Volatile chemical species released in the pyrolysis zone percolate through the char to the surface. The only effect on the virgin material is a small loss of volatiles. The backup or substrate material is unchanged by the effects of heat from the ablation process.

The ablation mechanism is assumed to be a surface chemical equilibrium, diffusion-controlled phenomena, where external combustion products react with the carbonaceous char and py-

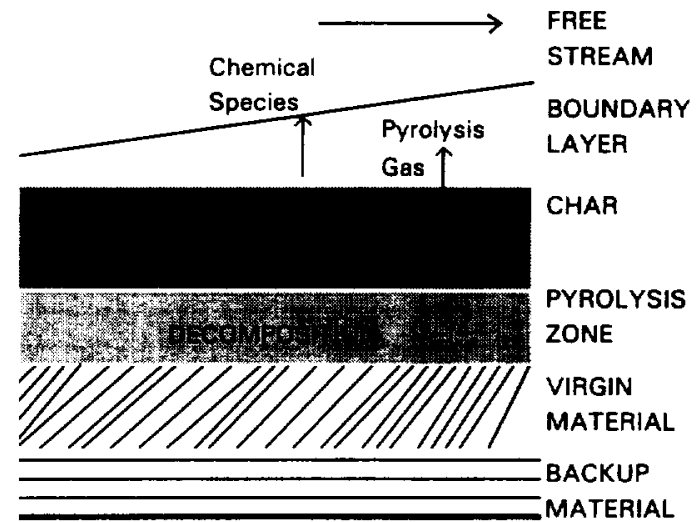

Fig. 1 Schematic of material ablation process model.

rolysis gases at an exponential rate. ${ }^{1.2}$ The ablation rate is proportional to the diffusion rate and concentration of reacting species that diffuse through the boundary layer. For pyrolysis of a composite material the resin filler is assumed to consist of two or more components that decompose separately (designated by $j$ and $k$ ), whereas the reinforcing fibrous material (designated by $l$ ) is the third component that can decompose. The instantaneous density of the composite is thus given by ${ }^{4}$

$$
\rho=\Gamma\left(\rho_{j}+\rho_{k}\right)+(1-\Gamma) \rho_{i}
$$

The volume fraction of the resin $\Gamma$ must be input into the simulation codes. It is assumed that each of the three components decompose according to the kinetic reaction described by

$$
\frac{\partial \rho_{m}}{\partial t}=-k_{m} \rho_{o m}\left(\frac{\rho_{m}-\rho_{r m}}{\rho_{o m}}\right)^{n}
$$

The decomposition rate constant $k_{m}$ must be accurately determined to calculate the material ablation rate.

\section{Thermogravimetric Analysis}

The material ablation process is quantified in terms of the conversion weight fraction $C$, which is the fraction of material already converted or decomposed at any instant in time. ${ }^{9.19}$ For a given sample of material, the conversion weight fraction is defined by

$$
C=\left(W_{o}-W\right) /\left(W_{o}-W_{r}\right)
$$

The decomposition rate of a material is a function of a temperature-dependent rate constant and the degree of conversion (or weight loss) , $^{8,19}$

$$
\frac{\mathrm{d} C}{\mathrm{~d} t}=k(T) f(C)
$$

The temperature is assumed to be a linear function of time and the specified heating rate

$$
T=q t+\text { const }
$$

The rate constant is assumed to be represented by the Arrhenius form ${ }^{8,14.20}$

$$
k(T)=A \exp (-E / R T)
$$

Substituting Eqs. (5) and (6) into Eq. (4) yields the fundamental kinetic model for the thermogravimetric decomposition rate $^{8,20,21}$

$$
\frac{\mathrm{d} C}{\mathrm{~d} T}=\frac{A}{q} f(C) \exp \left(-\frac{E}{R T}\right)
$$




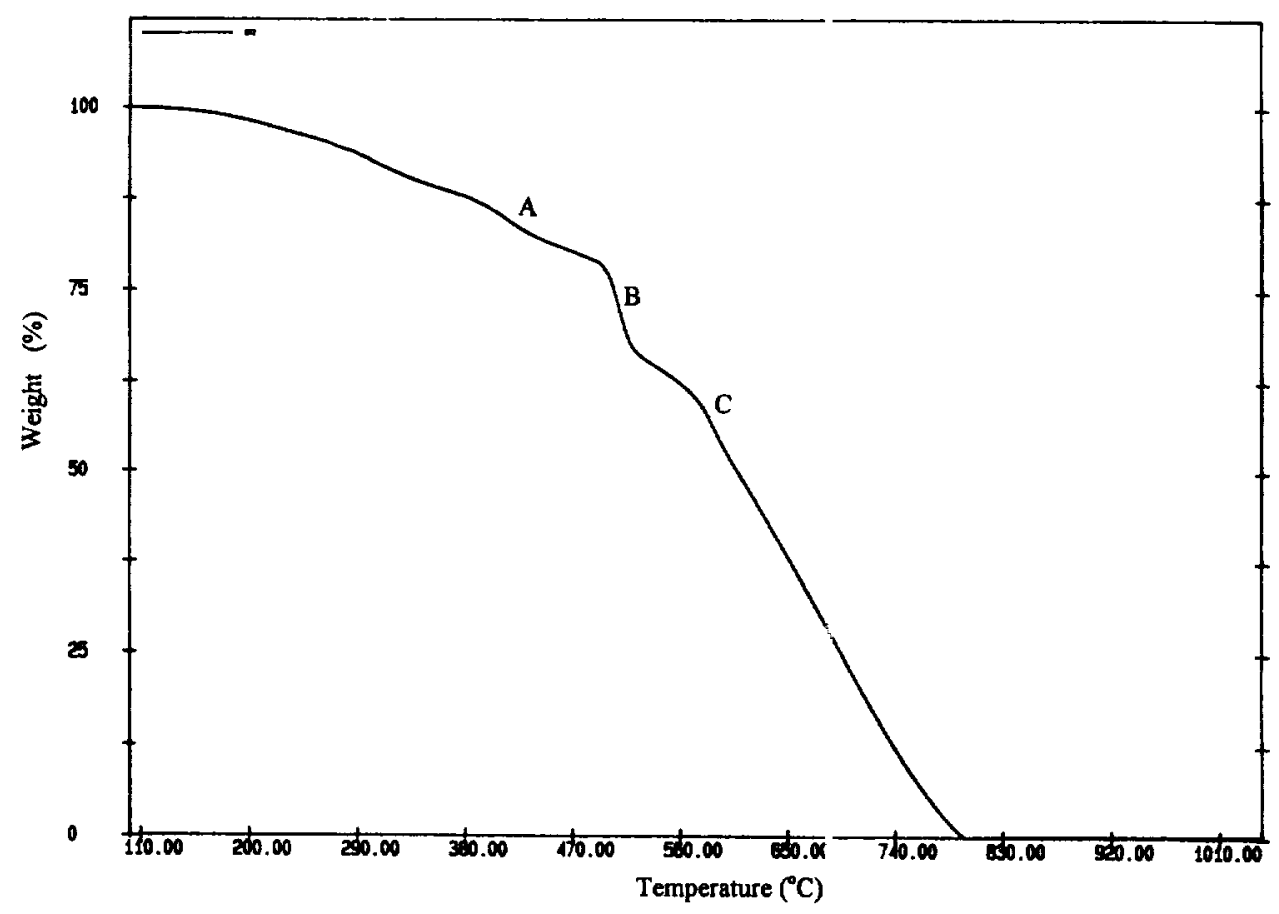

Fig. 2 TGA thermogram for phenolic resin, $q=20^{\circ} \mathrm{C} / \mathrm{min}$.

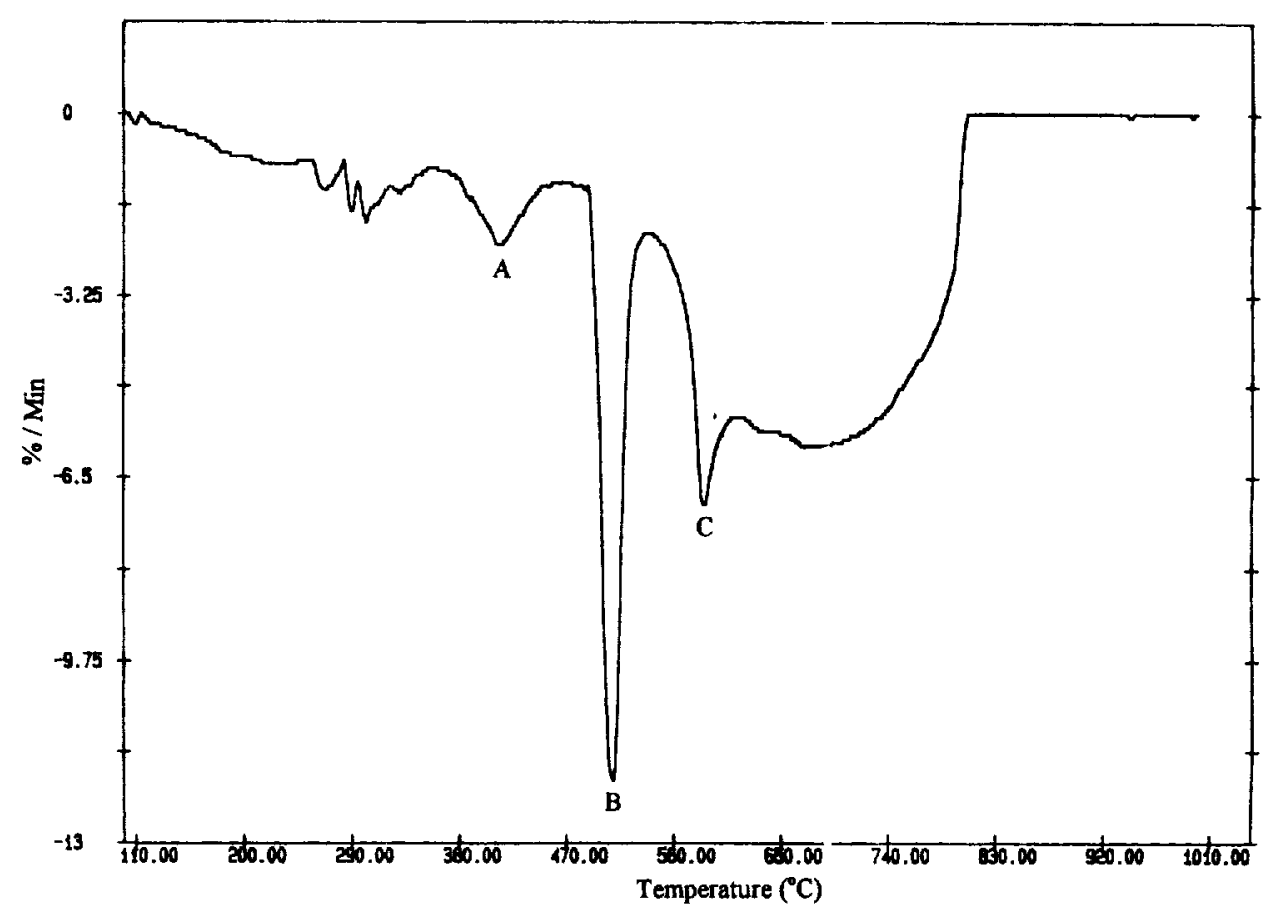

Fig. 3 Time derivative of phenolic resin thermog am shown in Fig. 2.

The TGA system monitors the weight of a sample as it is heated isothermally or at a specified heating rate. The isothermal approach is often avoided because it is impossible to instantaneously raise the material to the desired temperature before some initial weight loss occurs.' Therefore, the specified heating rate approach is generally used. The most common output from the TGA system is a thermogram, which is a plot of the percent of material remaining versus temperature. Figure 2 shows a typical thermogram for phenolic resin SC1008 heated at a rate of $20^{\circ} \mathrm{C} / \mathrm{min}$. Each region of slope change (indicated as A, B, and C) on the curve corresponds to an area of rapid weight loss. Typically, these areas represent different reactions ti king place in the material as it is heated. By taking the time di rivative of the curve, as plotted in Fig. 3, the different reac ions can be seen more clearly as inverted peaks.

If the re iction rate continues to decrease with decomposition, the rite of conversion is proportional to the amount of remaining condegraded material, ${ }^{9,14,19}$ described by

$$
f(C)=(1-C)^{n}
$$

Because reaction parameters are usually obtained with commercial ins?ruments from the beginning of the decomposition, 
a first-order reaction model is commonly assumed $(n=1),{ }^{22}$ and the decomposition rate may be expressed as

$$
\frac{\mathrm{d} C}{\mathrm{~d} T}=\frac{A}{q}(1-C) \exp \left(-\frac{E}{R T}\right)
$$

A quick, direct method for determining activation energy from TGA data was proposed by Flynn and Wall. ${ }^{14}$ They assumed that $A, f(C)$, and $E$ are independent of $T$, and that $A$ and $E$ are also independent of $C$. As discussed in Refs. 14 and 21 , separating variables and integrating Eq. (9) yields

$$
\begin{gathered}
F(C)=\int_{0}^{C} \frac{\mathrm{d} C}{(1-C)}=\frac{A}{q} \int_{T_{o}}^{T} e^{-E / R T} \mathrm{~d} T \\
F(C)=\left(\frac{A E}{q R}\right)\left[e^{-E / R T} /\left(\frac{E}{R T}\right)+\int_{-\infty}^{-E / R T}\left(\frac{e^{x}}{x}\right) \mathrm{d} x\right] \\
F(C)=\left(\frac{A E}{q R}\right) p(x)
\end{gathered}
$$

where $x=E / R T$. The integral in Eq. (10) cannot be evaluated directly and the value of $p(x)$ must be estimated. Taking the $\log$ of Eq. (10) yields

$$
\log [F(C)]=\log (A E / R)-\log (q)+\log p(x)
$$

Doyle $^{21}$ found that for $E / R T \geq 20, \log p(x)$ may be approximated at a constant percent conversion by

$$
\log [p(E / R T)] \cong-2.315-0.457 E / R T
$$

Doyle's calculations show that the value of the coefficient, 0.457 , does not vary more than $\pm 1 \%$ over the range $29 \leq E / R T \leq$ 42. Substituting Eq. (12) into Eq. (11) and differentiating at a constant percent of conversion, i.e., $C=$ const, yields

$$
\mathrm{d}(\log q)=\frac{-0.457 E}{R}\left[\mathrm{~d}\left(\frac{1}{T}\right)\right]
$$

Solving Eq. (13) for $E$ yields

$$
E=\frac{-R}{0.457}\left[\frac{\mathrm{d} \log (q)}{\mathrm{d}(1 / T)}\right]
$$

The bracketed term in Eq. (14) can be obtained from TGA data as the slope of a plot of $\log (q)$ versus $1 / T$ at a constant degree of conversion.

\section{Thermogravimetric Measurements}

\section{TGA Instrument}

A Perkin-Elmer TGA-7 instrument ${ }^{23}$ was used in this research to generate thermograms for the neat resin decomposition. This instrument records the thermal response of a material as it is heated at a specified heating rate. A typical TGA is shown schematically in Fig. 4. The TGA-7 primarily consists of an ultramicrobalance and a furnace element. Using a null-balance design the microbalance detects changes in weight as small as $0.1 \mu \mathrm{g}$. Its maximum capacity is $130 \mathrm{mg}$. According to the manufacturer balance accuracy is better than $0.1 \%$ of full scale. ${ }^{23}$ The furnace has the capability to operate from ambient temperature to $1000^{\circ} \mathrm{C}$ at the minimum linear heating rate of $0.1^{\circ} \mathrm{C} / \mathrm{min}$ and a maximum heating rate of $200^{\circ} \mathrm{C} / \mathrm{min}$ (in $0.1^{\circ} \mathrm{C} / \mathrm{min}$ increments). This feature accommodates the requirement of the Flynn and Wall method for a minimum of three distinct heating rates to calculate activation energy. ${ }^{14}$ Other major components of the system include a thermocouple, gas purge system, and a data-acquisition system.

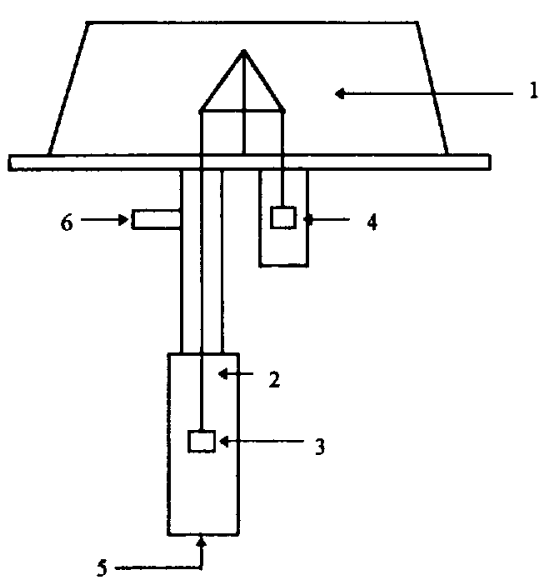

Fig. 4 Schematic of a typical TGA instrument. 1, balance; 2, furnace; 3 , sample; 4 , counterweight; 5 , gas inlet; and 6 , gas exit.

\section{Parameters Affecting Experimental Uncertainty of the TGA Process}

There are several sources of uncertainty in any TGA analysis. Among these are fluctuations in the balance, measurement of temperature by the thermocouple, and sample preparation. The weight measurements are affected by vibrations, static electricity, the thermocouple touching the balance pan, and purge-gas flow rate. From experiment trials and other studies, ${ }^{8,24-26}$ it was determined that these factors are relatively minor and controllable.

Because TGA samples are measured in milligrams, small sample sizes can potentially present a problem in terms of sample content homogeneity if proper precautions are not taken. With composite material the resin and fiber content may vary too widely in small samples. Samples for this investigation are pellets taken from a sheet of cured neat resin. Because no fibers are present sample homogeneity is less of a concern.

\section{Thermocouple Calibration}

A chromel-alumel thermocouple is employed for the TGA temperature measurement. Gradients between the sample and the thermocouple should be minimized or at least quantified. A Curie-point calibration ${ }^{21.23,24.27}$ was performed for each heating rate that was used. This calibration consists of heating from 2 to 5 ferromagnetic metal standards over a specified heating range at the desired heating rate. The standards are lowered into the furnace with an appropriately shaped magnet around it. Once heated these ferromagnetic metal standards lose their magnetism at a specific temperature and this loss in magnetism is manifested as an apparent weight loss that is measured by the TGA. In 1978 a study was conducted by the International Confederation for Thermal Analysis ${ }^{28}$ to evaluate the variance in standard temperature with instrument and technique. Eighteen different researchers using instruments from nine different manufacturers participated in the study. The bias in standard temperature was found to be markedly dependent upon magnet placement, temperature, and thermocouple location in a particular instrument. Based on these results TGA instrument manufacturers have since sought to minimize the bias as a result of this experimental technique with specific instructions on the placement of the magnet and thermocouple for their systems.

The temperature distribution within the TGA furnace also varies with heating rate. It has been observed that the variance associated with heating rate increases near the temperature limit of the instrument. Consequently, a calibration should be performed for each combination of thermocouple, sample holder, and heating rate in accordance with the test objectives. It is often recommended to perform the Curie calibration using two standards. ${ }^{23.29}$ However, four Curie standards were used for calibrating the TGA-7 in the research of this paper. Be- 
cause the TGA-7 instrument software only accepts two calibration values the two input values were taken from a linear regression that was based on the four calibration points. This was done to minimize error from a two-point fit and to allow calculation of the associated bias from the linear relationship between standard and thermocouple temperatures.

\section{Experimental Procedure}

For this study, 10 pellet samples of neat polymeric resin, Borden SC 1008 , were heated to $1000^{\circ} \mathrm{C}$ at each of three different heating rates of 5,10 , and $20^{\circ} \mathrm{C} / \mathrm{min}$. These heating rates were chosen based on manufacturer's recommendations. ${ }^{22}$ In addition, temperature errors associated with the use of pellets is minimized with low heating rates. Argon was used as the sample purge gas at a flow rate of $20 \mathrm{cc} / \mathrm{min}$. The samples were cured at $121^{\circ} \mathrm{C}$ and ambient pressure. To minimize errors caused by sample nonuniformity, variations in resin pellet weight were kept as small as possible. The TGA manufacturer recommended that the variance in sample weight be no more than $\pm 20 \%$. $^{23}$ For this study sample weights were held within \pm 0.6 of $10 \mathrm{mg}$ or $\pm 6 \%$. To eliminate moisture samples were held at $100^{\circ} \mathrm{C}$ for $10 \mathrm{~min}$ and then reweighed. This new dry weight was input to the TGA as a basis for the weight-loss thermograms. The amount of moisture per sample was less than $2 \%$ by mass. Data from seven previous trials of the same material at $20^{\circ} \mathrm{C} / \mathrm{min}^{30}$ were combined with this data set and used to calculate activation energy. Temperature data at each percent conversion for each thermogram were obtained by using TGA-7 data analysis software. The percent conversions chosen were based on a manufacturer's recommendation ${ }^{22}$ that data be taken from the beginning of the weight-loss curve. The material first starts to degrade at this point and practical material lifetime ends. ${ }^{23}$ It is this activation energy that is reported for this study. In addition, for the $\mathrm{SC} 1008$ resin used, activation energy could not be computed beyond $6 \%$ conversion. For this material, several reactions occur and other techniques would have to be employed to determine activation for each subsequent reaction. ${ }^{8,9}$

\section{Calculation of Activation Energy from TGA Data}

The variance in sample temperature with heating rate forms the basis of the calculation of activation energy by the Flynn and Wall technique. This approach, which is commonly used in the data reduction software of commercial TGA instruments, assumes a linear relationship between the ordinate $\log q$ and the abscissa $1 / T^{14}$ A linear least-squares method is used to determine the slope of this plot. This slope is input to Eq. (14) to determine activation energy.

However, in a typical TGA experiment, temperature is the experimental value that is actually being measured as opposed to heating rate that is essentially fixed. Therefore, temperature is the dependent variable, while heating rate is independent. By definition, the objective of the least-squares method is to determine the equation of the line that minimizes the vertical distance of each data point from that line. ${ }^{31.32}$ The ordinate (or uncontrolled variable) $y$ in an experiment depends upon the abscissa (or controlled variable) $x$ that has minimal variation. In the TGA experimental method temperature is obviously the uncontrolled variable and heating rate is controlled. Therefore, it would seem logical to minimize the variance in temperature rather than heating rate. Again, this is the opposite of the approach used in the software of many TGA manufacturers.

Rearranging Eq. (14) such that temperature is the dependent variable results in

$$
E=\frac{-R}{0.457\left[\frac{\mathrm{d}\left(\frac{1}{T}\right)}{\mathrm{d}(\log q)}\right]}
$$

The brack ted term in the denominator is the slope of a plot of $1 / T$ ver ius $\log (q)$. The activation energy was calculated by both the standard Flynn and Wall method [Eq. (14)] and modified meth od of Eq. (15). The modified method was used in the uncertiinty analysis.

\section{Experimental Uncertainty Analysis}

In this nvestigation the experimental uncertainty analysis procedures detailed by Coleman and Steele ${ }^{i 6.33}$ are employed. In this ap roach the overall uncertainty in an experimental result $r$ is lefined in terms of bias and precision uncertainties by

$$
U_{r}=\left(B_{r}^{2}+P_{r}^{2}\right)^{1 / 2}
$$

The bias tncertainty in the final result is an estimate of the fixed error within the experimental system that cannot be minimized by averaging the results of several trials. Precision uncertainty is a measure of the randomness or scatter in the data. This type of uncertainty arises as a result of measurement system precis: on errors and also variations in test conditions from trial to tria of the same experiment. The true result lies within the interva: $r \pm U_{r} 95 \%$ of the time.

The unc ertainty in activation energy was calculated based on the motified form of Eq. (15). Because Doyle's coefficient of 0.457 is not truly constant, the variable $P$ is substituted in its place. Thus, $E$ is a function of four experimental variables

$$
E=E(R, P, T, q)
$$

Following standard TGA procedures ${ }^{23}$ the final value of $E$ is assumed $\mathrm{tc}$ be the average value of activation energy at 1,3 , and $6 \%$ ccnversions, $\bar{E}_{136}$. Based on Eq. (15) the average activation en srgy is thus a function of the average of the slope of the $1 / T$ versus $\log (q)$ curve at 1,3 , and $6 \%$ conversion

$$
\bar{E}_{1: 5}=\frac{-3 R}{P\left(\mathrm{SLOPE}_{1 \%}+\mathrm{SLOPE}_{3 \%}+\mathrm{SLOPE}_{6 \%}\right)}
$$

There ar: a total of 111 different values of $T$, each measured at a specit ed corresponding heating rate. Therefore the full functional orm for the average activation energy is

$$
\bar{E}_{136}=f\left(R, P, T_{1}, \ldots T_{111}, q_{1}, \ldots q_{111}\right)
$$

Each expejimental parameter in $E$ has an associated bias represented by $B_{R}, B_{P}, B_{T}$, and $B_{q}$, respectively. Engineering constants are 1ypically known with greater accuracy than experimental par imeters and, thus, the bias in the gas constant $B_{R}$ is assumed ne gligible. $B_{P}$ is taken from Doyle ${ }^{21}$ as $\pm 1 \%$ of 0.457 . According to Perkin-Elmer $B_{q}$ is negligible. The bias in temperature, $h$ wwever, is not negligible. The elemental sources of bias consicered in the temperature measurements are $B_{\mathrm{CF}}$ as a result of curve fitting the thermocouple calibration data, and $B_{\mathrm{STO}}$ from he Curie standards. Following Ref. 16, $B_{\mathrm{CF}}$ is obtained fron

$$
B_{\mathrm{CF}}=2\left(S_{\mathrm{CF}}\right)
$$

where

$$
S_{\mathrm{CF}}=\left[\frac{1}{N-2} \sum\left(T_{\mathrm{CF}}-T_{\mathrm{CAL}}\right)^{2}\right]^{1 / 2}
$$

The value of $B_{C F}$ was calculated to be $7^{\circ} \mathrm{C}$. The bias in the standard was estimated to be of the same magnitude and is taken to be $7^{\circ} \mathrm{C}$. These elemental error sources are combined by the rss : nethod to obtain the total temperature bias as

$$
B_{T}=\left(B_{\mathrm{CF}}^{2}+B_{\mathrm{STD}}^{2}\right)^{1 / 2}=9.9^{\circ} \mathrm{C}
$$


It is also necessary to consider the effects of correlated terms on uncertainty. These terms arise when elemental errors in different measured variables originate from identical sources. For the TGA measurements of this study the temperature readings are correlated because each measurement of temperature is affected by the same bias uncertainties in the standards used in the calibration procedure. These covariance terms are approximated by the sum of products method. ${ }^{34}$

Considering the various sources of bias uncertainty relevant to this study the resultant bias limit in activation energy is

$$
\begin{aligned}
B_{E}^{2} & =\sum_{i=1}^{112}\left(\frac{\partial E}{\partial T_{i}}\right)^{2} B_{T_{i}}^{2}+\left(\frac{\partial E}{\partial P}\right)^{2} B_{p}^{2} \\
& +\sum_{i=1}^{111} 2\left(\frac{\partial E}{\partial T_{i}}\right) \sum_{j=i+1}^{112}\left(\frac{\partial E}{\partial T_{j}}\right) B_{T, r_{j}}
\end{aligned}
$$

A forward finite differencing method ${ }^{35}$ is used to calculate the partial derivative terms that are called sensitivity coefficients.

The precision limit $P_{r}$ specifies the confidence interval around a single result, where the biased mean of the experimental result lies $95 \%$ of the time. ${ }^{33}$ The precision uncertainty includes both correlated and uncorrelated terms. Although there has been one documented study that indicated correlated precision terms can be significant, ${ }^{36}$ these terms are typically assumed to be insignificant and are neglected in this paper. The precision uncertainty is estimated for large sample sizes of 10 or more. Also, the result of activation energy is averaged from several trials. Therefore, the precision uncertainty in the average activation energy is ${ }^{3.3}$

$$
P_{\bar{E}}=2\left(S_{E} / \sqrt{N}\right)
$$

where

$$
S_{E}=\left[\frac{1}{N-1} \sum_{i=1}^{N}\left(E_{i}-\bar{E}^{2}\right)\right]^{1 / 2}
$$

Based on the calculated bias and precision uncertainties, the uncertainty in the mean activation energy $\bar{E}$ is

$$
U_{\dot{E}}=\left(B_{E}^{2}+P_{\tilde{E}}^{2}\right)^{1 / 2}
$$

\section{Results and Discussion}

The effects of uncertainty in activation energy determined by TGA for the decomposition of a polymeric neat resin was assessed. Ten samples of neat resin (Borden SC1008) were heated to $1000^{\circ} \mathrm{C}$ for each of three different heating rates to obtain a thermogram of weight loss versus temperature. Activation energies obtained from the Flynn and Wall method and a modification of this method, where the ordinate and abscissa in the regression are reversed, were each examined. Additionally, uncertainties associated with the experimental approach were investigated. Finally, the effect of uncertainty in activation energy on the ablation rate for a composite solid rocket nozzle was also investigated.

A Fortran program was developed to calculate activation energy by both the traditional Flynn and Wall and modified methods. ${ }^{37}$ The program was structured to read temperature data for all three heating rates, convert to the appropriate units, and store the converted data in an array. The converted data were initially used to calculate $E$ for each percent conversion based on Eq. (14). Activation energies calculated by the Fortran program were compared to the $E$ values calculated by the Perkin-Elmer instrument software. These values generally agreed to within $\pm 3 \mathrm{~kJ} / \mathrm{g}$-mol or $5 \%$. Therefore, the activation energies presented are those calculated by the Fortran program and not the Perkin-Elmer software. The Fortran program calculates the partial derivatives with respect to each temperature variable and Doyle's coefficient defined in Eq. (23) by a for- ward finite differencing technique. From the partial derivatives the systematic and correlated bias uncertainties were then calculated by Eqs. (23-25). The total experimental uncertainty in $E$ was then determined by Eq. (26).

\section{Activation Energy Calculation}

The Flynn and Wall method has been widely used in TGA instrumentation to calculate activation energy. This method utilizes the relationship between the log of the heating rate and reciprocal temperature to derive an equation for activation energy. According to Flynn and Wall ${ }^{14}$ this relationship can best be described by a linear mathematical model. A summary plot of $\log (q)$ versus $1 / T$ data for various percent conversions is shown in Fig. 5. A linear regression method was used to determine the slope at each percent conversion. The sample $r$ is a dimensionless quantity that describes the degree of linear association between two variables. ${ }^{35}$ For the curve-fits in Fig. $5, r$ ranges from 0.91 to 0.60 for conversion percentages of $1-6 \%$, respectively. The calculated slopes are input to Eq. (14) to determine $E$.

In a TGA experimental setting the heating rate is fixed and the temperature is the observed response that is being measured. By definition, the ordinate (uncontrolled) variable in an experiment depends upon the abscissa (controlled) variable. The objective of the least-squares method is to determine the equation of the line that minimizes the vertical distance of each data point from the regression line. ${ }^{31.32}$ There is a negligible variation in heating rate throughout a specified heating profile. However, temperature, for a particular percent conversion at a fixed heating rate, varies slightly as shown in Fig. 6 for $1 \%$ conversion. Comparable variations in temperature were observed for each of the other percent conversions. Because this scatter is present in the temperature data, the effect of inter-

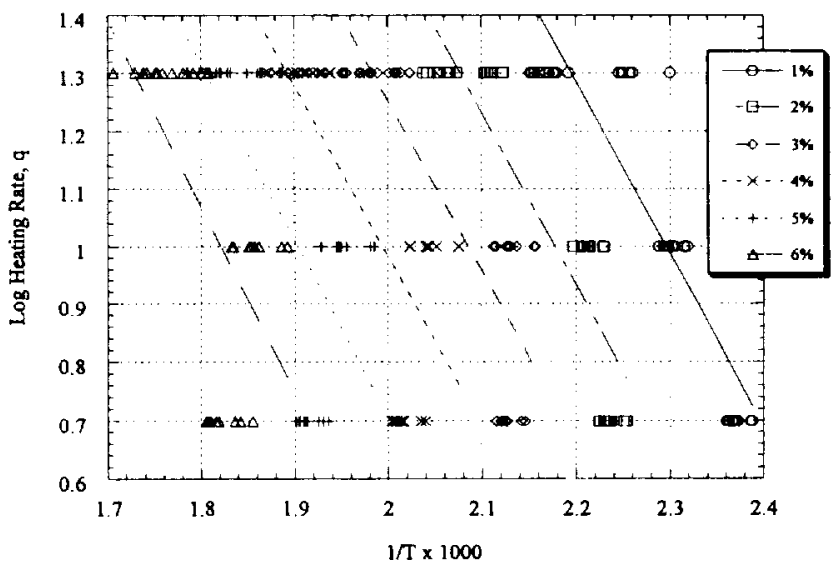

Fig. 5 Plot of $\log (q)$ versus $1 / T$ for neat resin.

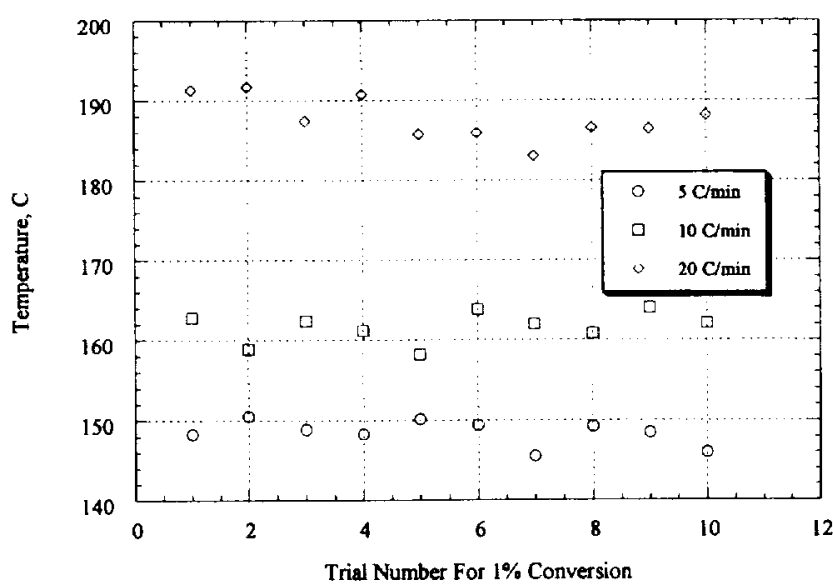

Fig. 6 Scatter in TGA temperature data for $1 \%$ conversion. 
changing the abscissa $x$ and ordinate $y$ in Fig. 5 was examined. Figure 7 is a plot of $1 / T$ versus $\log (q)$. The sample $r$ for this plot also ranges from 0.91 to 0.60 for percent conversions of $1-6 \%$, respectively. Linear regression is again used to determine the slopes that are inserted in the bracketed term in Eq. (15) to calculate $E$ at each percent conversion.

Table 1 lists the values of $E$ from 1 to $6 \%$ conversion calculated with the Fortran program and both methods. Activation energies calculated with the standard Flynn and Wall approach [Eq. (14)] vary from 54 to $59 \mathrm{~kJ} / \mathrm{g}$-mol with a mean of $55 \mathrm{~kJ} /$ $\mathrm{g}$-mol. These values were used to calculate the respective values of $E / R T$ and verify the assumptions used to derive Eq. (14). The observed variation in $E / R T$ insignificantly affected Doyle's constant. Using the modified Flynn and Wall method [Eq. (15)] the calculated activation energies vary from 66 to $160 \mathrm{~kJ} / \mathrm{g}$-mol with a mean value of $96 \mathrm{~kJ} / \mathrm{g}$-mol. Therefore, using the modified method produced a much larger variation in $E$ and a $75 \%$ larger mean value. A detailed comparison of the regression statistics revealed that both methods had identical sample $r$ values at the same percent conversions even though the actual values of $E$ were significantly different. For example, at $1 \%$ conversion the correlation coefficient for both methods is 0.91 , but $E$ determined with the modified method is $20 \%$ greater than that for the standard method. At $6 \%$ conversion $r=0.6$ and the $E$ calculated by the modified method is approximately 2.7 times larger than the value calculated with the standard method. However, even with a relatively low sample correlation coefficient of 0.6 for $6 \%$ conversion, the largest $E$ obtained by Eq. (14) is within $4 \mathrm{~kJ} / \mathrm{g}-\mathrm{mol}$ of the mean. Using the modified method the variation in $E$ from 1 to $6 \%$ conversion is much larger relative to the mean value. However, more significantly, the standard errors for the modified method were a factor of 3-4 times smaller than the errors calculated for the standard method. This would indicate that the modified method produces a more accurate curve fit of the TGA data. The larger variation in $E$ over the $1-6 \%$ conversions may also indicate that the modified method is more sensitive to nonlinearity of

Table 1 Experimental activation energies for $\mathrm{SC1008}$ neat resin

\begin{tabular}{|c|c|c|}
\hline \multirow[b]{2}{*}{ Percent conversion, $\%$} & \multicolumn{2}{|c|}{ Activation energy, $\mathrm{kJ} / \mathrm{g}-\mathrm{mol}$} \\
\hline & Eq. (14) & Eq. (15) \\
\hline 1 & 54.0 & 65.5 \\
\hline 2 & 54.4 & 67.9 \\
\hline 3 & 53.7 & 74.8 \\
\hline 4 & 53.6 & 91.4 \\
\hline 5 & 55.6 & 113.5 \\
\hline 6 & 58.6 & 159.8 \\
\hline Average $1-6 \%$ & 55.0 & 95.5 \\
\hline Average 1,3 , and $6 \%$ & 55.5 & 100.0 \\
\hline
\end{tabular}

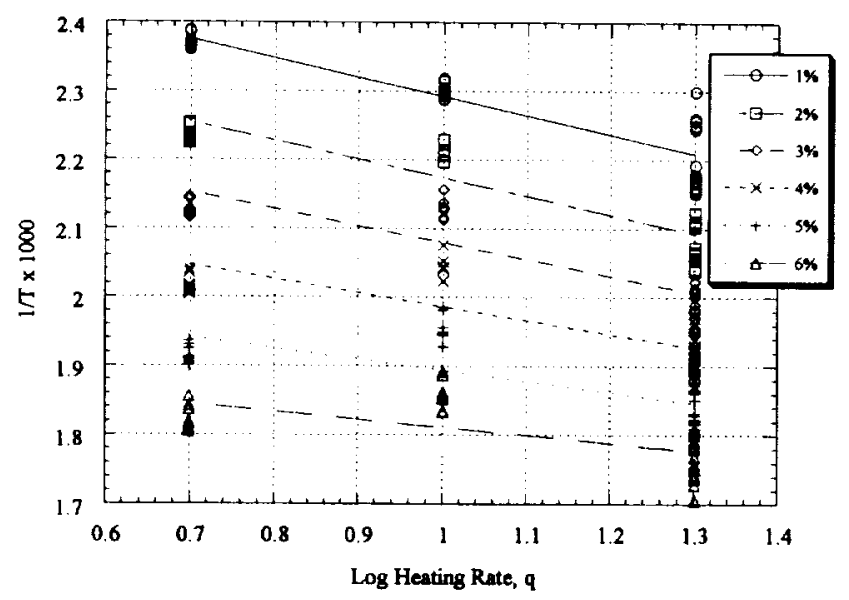

Fig. 7 Plot of $1 / T$ versus $\log (q)$ for neat resin. the TGA dita. This nonlinearity is possibly an indication that the assumpion of a first-order decomposition reaction is not an accurate representation for this resin.

In practice, the final activation energy is the average of the activation $t$ nergies at three different percent conversions. In this research, 1,3 , and $6 \%$ conversions were chosen to calculate the a verage activation energy. The final average $E$ based on these va ues was $56 \mathrm{~kJ} / \mathrm{g}$-mol using the standard Flynn and Wall metho $J$ and $100 \mathrm{~kJ} / \mathrm{g}$-mol using the modified method.

\section{Uncertainty in Activation Energy and Its Effect} on Ablation Rate

The modified method [Eq. (15)] and Eqs. (23-26) were used to determine the uncertainty in the calculated average activation energy. The resultant precision $P_{\bar{E}}$, bias $B_{\bar{E}}$, and total uncertainties $U_{\bar{E}}$ are, respectively, $60.0,16.2$, and $60.1 \mathrm{~kJ} / \mathrm{g}-\mathrm{mol}$. The overall uncertainty of $60.1 \mathrm{~kJ} / \mathrm{g}-\mathrm{mol}$ is $60 \%$ of the average $E$ of $100 \mathrm{k} / \mathrm{g}$-mol.

These results were used to evaluate the effect of uncertainty in activation energy on the ablation rate. The CMA92FLO code was ured to determine the thermal response of a carbonphenolic nczzle throat exposed to propellant gases without pore-pressure calculations. A baseline case was first run with a standard input value for $E$. Although the uncertainty analysis yielded a $60 \%$ uncertainty in $E$, Table 1 shows a much larger variation in activation energy from 1 to $6 \%$ conversion. Therefore, as a conservative estimate of uncertainty effects, the CMA code was rerun with the activation energies doubled for each of the three components of degrading material. The calculated ablation rates for the baseline and doubled-activation energy cases were compared after $30 \mathrm{~s}$ of a simulated solid rocket motcr firing. Doubling the activation energy increased the ablation rate by $3 \%$. Depending on several factors, a $3 \%$ increase in surface recession could be significant. Operating conditions if the motor could cause the effect of uncertainty in activatio 1 energy to increase or decrease. In addition, changes in 'ther input parameters such as enthalpy could increase or decrease the impact of activation energy uncertainty on ablation.

\section{Summary, Conclusions, and Recommendations}

Kinetic $r \in$ action parameters are used as inputs to computer codes that $\mathrm{p}$ redict rocket nozzle ablation. The kinetic reaction parameter $o$. activation energy is typically obtained from TGA data. A com prehensive literature search did not indicate that anyone had systematically assessed the experimental uncertainty associated with the final determination of activation energy by one particular methodology and commercially available instrum entation. The study described in this paper was conducted tc characterize the uncertainty in $E$ and to determine if this uncer ainty had any affect on the predicted surface recession rate of a simulated composite solid rocket nozzle. Carbon-phenolic material was originally considered for this research; ho wever, most data for this class of composites are restricted. T lerefore, a neat resin, Borden $\mathrm{SC1008}$, was used. The uncertanty in activation energy of a composite may be greater than that of a neat resin because of errors caused by sample nont niformity. Temperature and weight-loss data were gathered at reating rates of 5,10 , and $20^{\circ} \mathrm{C} / \mathrm{min}$. Activation energy was determined from these data. The research methodology wa; derived from established experimental uncertainty methc ds, standard TGA operating procedures, experimental data, and a literature review.

Two meth $\mathrm{d}$ s for calculating activation energy were examined: 1) the traditional Flynn and Wall method based on the slope of $\log (1)$ versus $1 / T$ and 2 ) a modification of this method where the ordinate and abscissa are reversed. The Flynn and Wall approach assumes heating rate is the random variable. In reality the heating rate is essentially fixed and scatter is observed in the temperature data. Traditional methods of calculating $E$ from TGA data are actually inverse regression esti- 
mations. Therefore, a modified method was employed in which activation energy is calculated with the slope determined from a plot of $1 / T$ versus $\log (q)$. The modified method complies more closely with standard linear regression principles and produced a much larger variation in $E$ over $1-6 \%$ conversion than for the standard method. Because of this variation the average value of activation energy using the modified method was $75 \%$ larger than the average value using the standard method. But detailed regression statistics show that the modified method actually reduces the standard error at each percent conversion by a factor of 3-4 compared to the standard Flynn and Wall method. Therefore, the modified Flynn and Wall approach appears to produce a more accurate curve fit of the TGA data and is more sensitive to data nonlinearity.

A detailed uncertainty analysis was performed based on the modified method. The average activation energy for 1,3 , and $6 \%$ conversion $\left(\bar{E}_{136}\right)$ was calculated to be $100 \mathrm{~kJ} / \mathrm{g}$-mol with an uncertainty $\left(U_{\bar{E}}\right)$ of $60 \mathrm{~kJ} / \mathrm{g}$-mol. Based on this result, standard activation energies for a carbon phenolic material were increased by a factor of 2 and used to calculate the ablation rate in a typical solid rocket environment. Doubling the standard activation energy for a carbon-phenolic increased the predicted recession rate in a typical solid rocket environment by $3 \%$. A $3 \%$ increase in surface recession could be significant depending on actual operating conditions of the motor and changes in other input parameters such as enthalpy.

This research indicates that when linear regression is used to obtain information from experimental data, significantly different results are obtained, depending on which variable is considered the dependent or random variable. This is particularly true for determination of activation energy from TGA data. Contrary to the approach used in the software of many TGA manufacturers, the activation energy should be calculated by a modification of the Flynn and Wall method using the slope determined from a plot of $1 / T$ as the ordinate versus $\log (q)$ as the abscissa.

\section{Acknowledgment}

The authors thank James Thompson, Alabama A\&M University, for providing access to the thermogravimetric analyzer.

\section{References}

'Bunker, R., and Prince A., "Hybrid Rocket Motor Nozzle Material Predictions and Results," AIAA Paper 92-3591, July 1992.

${ }^{2}$ Ikeda, H., Yamamoto, Y., and Kohno, M., "A Study of Erosion and Ablation Mechanisms in a Solid Rocket Motor Exit Cone," Proceedings of the 16th International Symposium on Space Technology and Science, Vol. 1, AGNE, Tokyo, Japan, 1988, pp. 191-196.

${ }^{3}$ Farmer, R. W., "Phenolic Resin Char-Formation During Hyperthermal Ablation," Thermochim Acta, Vol. 4, March 1972, pp. $223-$ 238 .

${ }^{4}$ Baker, D. L., Wool, M. R., and Schaefer, J. W., "A Dynamic Technique for Determining the Thermal Conductivity of Charring Materials," Proceedings of the 8th Conference on Thermal Conductivity, Plenum, New York, 1969, pp. 823-836.

'Clayton, W., Fabish, T., and Denman, G., "Effect of Ablation on Thermal Conductivity to $5000^{\circ} \mathrm{R}$ in Phenolic Carbon and Phenolic Graphite," U.S. Air Force Contract AF 339(615)-3804, Battelle Memorial Inst., Columbus, $\mathrm{OH}, 1970$

${ }^{6}$ Boyle, M. E.. and Cozzens, R. F., "The Effect of High Heating Rate on the Pyrolysis of Carbon/Phenolic Composites," U.S. Naval Research Lab., Rept. 6343, Washington, DC, Sept. 1988.

'Wendlandt, W. W., Thermal Analysis, Wiley, New York, 1986.

${ }^{8}$ Flynn, J. H., and Wall, L. A., "General Treatment of the Thermogravimetry of Polymers," Journal of Research, Vol. 70A, No. 6 , National Bureau of Standards, Aug. 1966, pp. 487-523.

${ }^{9}$ Nam, J., and Seferis, J., "A Composite Methodology for Multistage Degradation of Polymers," Journal of Polymer Science Part B: Polymer Physics, Vol. 30, 1992, pp. 455-463.
${ }^{10}$ Farmer, R. W., "Thermogravimetry of Phenol-Formaldehyde Polycarbonates: Part III. Carbon/Graphite Cloth Reinforced Composites to $1400^{\circ} \mathrm{C}$," Air Force Materials Lab., TR-65-246, Pt. III, Wright-Patterson AFB, OH, June 1970.

"Russell, G. W., "Evaluation of Decomposition Kinetic Coefficients for a Fiber-Reinforced Intumescent-Epoxy," AIAA Paper 931856, June 1993.

${ }^{12}$ Tant, M. R., Henderson, J. B., and Moore, G. R., "Thermogravimetric Determination of Kinetic Parameters for the Thermal Degradation of Several Ablative Materials," Naval Surface Weapons Center, TR 80-290, Dahlgren, VA, Dec. 1980.

${ }^{13}$ Zsako, J., "Kinetic Analysis of Thermogravimetric Data, VI, Some Problems of Deriving Kinetic Parameters from TG Curves," Journal of Thermal Analysis, Vol. 5, No. 1, 1973, pp. 239-251.

${ }^{14}$ Flynn, J. H., and Wall, L. A., "A Quick, Direct Method for the Determination of Activation Energy from Thermogravimetric Data," Polymer Letrers, Vol. 4, 1966, pp. 323-328.

${ }^{15}$ Stokes, E., "Kinetics of Pyrolysis Mass Loss from Cured Phenolic Resin," AIAA Paper 94-3182, June 1994

${ }^{16}$ Coleman, H. W., and Steele, W. G., Experimentation and Uncertainty Analysis for Engineers, Wiley, New York, 1989.

${ }^{17}$ Brown, K, "Assessment of the Experimental Uncertainty Associated with Regressions," Ph.D. Dissertation, Univ. of Alabama in Huntsville, AL, 1996.

${ }^{18 . " A r m s ~ a n d ~ M u n i t i o n s, ~ C r i m e, ~ E x p o r t s, ~ P e n a l t i e s, ~ S e i z u r e s ~ a n d ~}$ Forfeitures," International Traffic in Arms Regulations, 22 Code of Federal Regulations Part 127, Government Printing Office, Washington, DC, July 1993.

${ }^{19}$ Zsako, J., "Kinetic Analysis of Thermogravimetric Data," Journal of Physical Chemistry, Vol. 72, No. 7, 1968, pp. 2406-2411.

${ }^{20}$ Zsako, J." and Zsako J., Jr. "Kinetic Analysis of Thermogravimetric Data," Journal of Thermal Analysis, Vol. 19, Jan. 1980, pp. $333-345$.

${ }^{21}$ Doyle, C. D., "Kinetic Analysis of Thermogravimetric Data," Journal of Applied Polymer Science, Vol. 5, No. 15, 1961, pp. 285292.

22."Instructions for the PC Series TGA 7 Decomposition Kinetics Software Kit," Perkin Elmer Corp., Norwalk, CT, Nov. 1991.

${ }^{23}$ "TGA 7 Thermogravimetric Analyzer Operator's Manual," Perkin Elmer Corp., Norwalk. CT, Nov. 1991.

${ }^{24}$ Blazek, A., Thermal Analysis, Van Nostrand Reinhold Co., London, England, UK, 1972.

${ }^{25}$ Newkirk, A., "Thermogravimetric Measurements," Analytical Chemistry, Vol. 32, No. 12, 1960, pp. 1558-1563.

${ }^{26}$ Earnest, C. M., "The Modern Thermogravimetric Approach to the Compositional Analysis of Materials," Compositional Analysis by Thermogravimetry, American Society for Testing and Materials, STP 997, 1988.

${ }^{27}$ Divito, M. P., Brenan, W. P., and Fyans, R. L., "Thermal Analysis: Trends in Industrial Applications," American Laboratory, Vol. 18, No. 1. 1986, pp. 82-95.

${ }^{28}$ Garn, P., Menis, O., and Wiedemann, H., "ICTA Certified Reference Materials for Thermogravimetry," U.S. National Bureau of Standards, GM-761, Gaithersburg, MD, 1978

29" Instruction Manual-951 Thermogravimetric Analyzer (TGA)," DuPont Corp., Wilmington, DE, 1988.

${ }^{30}$ Darby, S. P., and Landrum, D. B., "Experimental Uncertainty In Determining Kinetic Reaction Parameters for Polymeric Materials,' AIAA Paper 94-2088, June 1994.

${ }^{31}$ Haberman, R., Elementary Applied Partial Differential Equations, Prentice-Hall, Englewood Cliffs, NJ, 1983.

${ }^{32}$ Montgomery, D. C., and Peck, E. A., Introduction to Linear Regression Analysis, 2nd ed., Wiley, New York, 1992.

${ }^{33}$ Coleman, H., and Steele, W., "Engineering Application of Experimental Uncertainty Analysis," AIAA Journal, Vol. 33, No. 10, 1995, pp. $1888-1896$

${ }^{34}$ Brown, K.. Coleman, H., Steele, W., and Taylor, R., "Evaluation of Correlated Bias Approximations in Experimental Uncertainty Analysis," AIAA Journal, Vol. 34, No. 5, 1996, pp. 1013-1018.

${ }^{35}$ Wapole, R., and Myers, R., Probability and Statistics for Engineers and Scientists, 5th ed., Macmillan, New York, 1993.

${ }^{36}$ Hudson, S., Bordelon, W., and Coleman, H., "Effect of Correlated Precision Errors on the Uncertainty of a Subsonic Venturi Calibration," AIAA Journal, Vol. 34, No. 9, 1996, pp. 1862-1867.

${ }^{37}$ Darby, S. P., "Assessment of Uncertainty in the Determination of Kinetic Reaction Parameters for Polymeric Materials," M.S. Thesis, Univ. of Alabama in Huntsville, AL, 1997. 


